\begin{tabular}{llllllllllllllllllllllllllllllll}
\hline$R$ & $E$ & $V$ & I & S & T & A & D & E & E & S & T & U & D & I & O & S & I & N & T & E & $R$ & N & A & C & I & O & N & A & L & E & S
\end{tabular}

\title{
El entendimiento sobre el Tratado de Libre Comercio entre China y Chile
}

\author{
Zhang Xinsheng
}

Las relaciones chino-chilenas se encuentran actualmente en la mejor época de su historia, y al mismo tiempo, enfrentan una oportunidad importante para profundizarlas. Teniendo presente la situación del mundo y de la Cuenca del Pacífico, así como las relaciones chino-chilenas, ambos países resolvieron que era importante establecer mecanismos económico-comerciales bilaterales, y en junio de 2002, la República Popular China propuso a Chile iniciar negociaciones orientadas a suscribir un tratado de libre comercio, las que se formalizaron en noviembre de 2004, durante la cumbre de APEC. Tras 5 rondas de negociaciones, el 18 de noviembre de 2005 se firmó oficialmente el Tratado de Libre Comercio entre ambos países. Este importante paso puede reforzarse en el futuro aprovechando la creciente globalización económica, los avances de la ciencia y de la tecnología, las nuevas facilidades de transferencia de información y de comunicaciones y transporte, y afianzando el intercambio político y entre diversos sectores sociales de ambos países, a fin de profundizar el conocimiento y el entendimiento recíprocos. Para profundizar aún más esta relación se hace por tanto necesario, mantener intercambios entre las esferas políticas de ambos países; reforzar el intercambio entre los diversos sectores sociales; iniciar y completar cuanto antes las negociaciones sobre servicios e inversión al mismo tiempo, hay que reglamentar las relaciones económico-comerciales bilaterales en el marco del tratado de libre comercio, y reforzar la cooperación bilateral dirigida a terceros países, para que los efectos de estas se amplíen hacia el resto de los países de Sudamérica y Asia; explorar y probar nuevos modelos y formas de cooperación chino-chilena; y explotar las potencialidades de las empresas privadas de China, a fin de que participen en los esfuerzos por afianzar relaciones mutuas, incluso en las microempresas y las empresas pequeñas, medianas y grandes. 
118 de noviembre de 2005, en Corea, durante de la cumbre del APEC y al cumplirse el primer aniversario del inicio de las negociaciones por los presidentes de China y Chile, y en presencia de ambos, se firmó el Tratado de Libre Comercio entre China y Chile. El tratado solo abarca el comercio de productos y ambos países acuerdan el pronto comienzo de las negociaciones sobre el comercio de servicios e inversiones. El instrumento es muy importante para las relaciones bilaterales, y revela el deseo de promover aún más la cooperación entre países que se encuentran a ambos lados del Pacífico, y que tienen a la vez muchas similitudes y grandes diferencias.

\section{LA SITUACIÓN MUNDIAL Y LAS ACTUALES RELACIONES BILATERALES ENTRe China y ChILE FUERoN} FAVORABLES AL A LA CELEBRACIÓN DE

UN TRATADO DE LIBRE COMERCIO ENTRE AMBOS PAÍSES

Desde los años 80 y 90, las regiones de Asia oriental, incluida China, y América Latina y del Norte, incluido Chile, fueron nuevos puntos de desarrollo económico y de promoción del crecimiento económico del mundo. La globalización arrastra el crecimiento económico de los países situados alrededor del Pacífico, y al mismo tiempo que estos países profundizan reformas económicas y promueven con mucho esfuerzo la apertura hacia el exterior, sienten que hay que desarrollar relaciones económicas y comerciales con todos los países del mundo mediante la creación de un medio internacional favorable al desarrollo socioeconómico de cada uno de ellos. Sin embargo, el sistema mundial de comercio dista mucho de haberse perfeccionado: la nueva ronda de Doha de negociaciones comerciales mundiales tarda en alcanzar los resultados esperados y al mismo tiempo que se profundiza la globalización, la regionalización económica y la creación de un creciente número de mecanismos comerciales bilaterales y regionales se convierten en nuevas opciones para diversos países.

\section{El sistema mundial de comercio dista mucho de haberse perfeccionado.}

Desde que establecieron relaciones diplomáticas en 1971, y especialmente desde los años 90 del siglo pasado, las relaciones políticas y económicas bilaterales entre China y Chile, se han desarrollado de manera notable. Entretanto, en los primeros años del nuevo siglo, el gran avance de las relaciones bilaterales ha llamado atención de la sociedad internacional; Chile fue el primero país de América Latina que estableció relaciones diplomáticas con China, el primero de la región que reconoció la posición de esta en el comercio mundial y el primero en firmar un tratado de libre comercio con China. En 2001, cuando visitó Chile Jiang Zemin, a la sazón presidente de China, ambos presidentes declararon que las relaciones bilaterales eran «relaciones hacia el siglo 21 de perseverancia, estabilidad, igualdad y beneficio mutuo». Y en 2004, con motivo de 
la visita del presidente Hu Jintao, los presidentes de ambos países decidieron redefinir las relaciones mutuas como relaciones de «socios cooperativos en todos los aspectos», demostrando así que tanto las autoridades chinas como los gobiernos de Chile han prestado gran atención a sus vínculos recíprocos. En los últimos 5 años, el comercio entre China y Chile ha tenido un crecimiento anual promedio de dos dígitos, y en 2004 creció 51.9\% y, de acuerdo con cifras proporcionadas por la Aduana de China, las exportaciones de Chile a ese país aumentaron $63.5 \%$, mientras que de enero a octubre de 2005, el comercio bilateral incrementó $36 \%$, y su volumen total superó el alcanzado en todo 2004. Ese año China llegó a ser el segundo socio comercial de importancia para Chile después de los Estados Unidos, mientras que Chile es el tercer socio comercial más importante de China en América Latina.

\section{En 2004, China llegó a ser el segundo socio comercial de importancia para Chile.}

Las grandes complementariedades económicas y el vasto espacio de cooperación entre China y Chile constituyen un importante potencial para desarrollar las relaciones económico-comerciales entre ambos en el futuro. Para China, país de enorme población, cuya economía es de grandes alcances, y cuyo sistema económico incluye variados sectores económicos que se caracterizan por vastas diferencias entre las diversas regiones y que al mismo tiempo carece de suficientes recursos naturales per cápita, tiene grandes necesidades de recursos naturales, así como de productos agropecuarios y de la pesca. En cambio, Chile, pese a que su economía es relativamente pequeña, tiene una elevada competitividad internacional y, sobre todo, cuenta con cobre y otros productos mineros y agrícolas muy necesarios para China, lo que demuestra la complementariedad económico-comercial entre ambos. En China, aparte de las zonas oriental y costera en que los sectores industrial, comercial, financiero y de servicios están muy desarrolladas, tiene amplias zonas interiores cuyo grado de desarrollo es muy inferior y necesitan con urgencia capital, tecnologías y recursos humanos. Además, es muy necesario reformar las viejas bases industriales de las provincias del noreste de China, que pueden ser grandes espacios donde pueden operar las empresas chilenas. La economía de China se destaca por las diferencias regionales, ya que las diversas regiones del país tienen sus propias especialidades económicas. Por ejemplo, las economías del delta del río de las Perlas, del río Yang Tse y de las regiones que rodean el mar Bohai tienen distintas características y necesidades, que podrían ser estudiadas y explotadas por empresas chilenas. Pese a que sigue siendo un país en desarrollo, China cuenta con enorme capacidad de renovación y un elevado nivel científico y tecnológico, incluso en campos como los relacionados con la tecnología espacial, biológica, etc., que ofrecen amplio espacio para la cooperación científico-tecnológica. Tras un largo período de alto crecimiento económico, China se 
relaciona cada vez más con los diversos países y regiones del mundo, y está a punto de entrar en una etapa intensa de inversión en el exterior, gracias a que el gobierno está aplicando desde hace tiempo una política de inducir a las empresas locales a «marchar hacia el exterior», y aprovechar «los mercados y los recursos del interior y del exterior». El 15 de enero de 2006, el Banco Popular de China (Banco Central) informó que hasta fines de diciembre de 2005, el ahorro de la población de China se elevó a 14,928.1 billones de yuan de Renminbi (cerca de 2 billones de dólares norteamericanos), y que sus reservas internacionales habían llegado a 818.9 billones de dólares de los Estados Unidos, valores, que podrían ser fuentes de recursos financieros para Chile y otros países latinoamericanos.

\section{La magnitud de las economías y su comercio con el exterior son marcadamente asimétricas.}

Todo lo anterior habla de los aspectos positivos de las relaciones entre China y Chile; sin embargo, hay grandes diferencias entre ambos. Primero, existe una gran asimetría en cuanto a la magnitud de ambas economías y a su comercio con el exterior, lo que indica la importancia que representan para ambas partes las relaciones económico-comerciales bilaterales. Por una parte, China, cuyo producto interno bruto en 2005 fue 18,232.1 billones de yuan de Renminbi (cerca de 2,300 billones de dólares de los Estados Unidos), y cuyos principales socios económico-co- merciales son los Estados Unidos, Japón y Europa, presta cada vez más atención a las relaciones de cooperación con América Latina, pero no tiene posibilidades de que Chile sea un socio importante desde el punto de vista global. En 2005, el volumen total del comercio exterior de Chile fue de 85 mil millones de dólares, mientras que el de China superó los 1,400 billones de dólares. El comercio bilateral entre China y Chile fue de 5,360 millones de dólares. Estas grandes diferencias entre las economías y el comercio exterior de ambos países indican que Chile no podrá figurar entre los socios económicocomerciales más importantes de China. Y al revés, China, con su alto crecimiento económico, que promueve cada vez más abiertamente el crecimiento de Chile y de otros países latinoamericanos, es y será uno de los socios económico-comerciales más importantes de Chile, situación de la cual puede derivar una asimetría en cuanto a la actitud positiva de ambas partes respecto de la promoción de relaciones bilaterales de cooperación, y a pesar de que las autoridades de ambos gobiernos son partidarias de profundizar la cooperación bilateral, falta intercambiar información, conocimientos y personas de diversos estratos sociales entre ambos, como ser entre las empresas, y los estratos populares.

Las relaciones chino-chilenas atraviesan actualmente por el mejor período de su historia, y al mismo tiempo, enfrentan una oportunidad importante de desarrollo. Para profundizar aún más las relaciones de cooperación entre ambos es preciso superar algunos problemas, tales como el hecho de que gran parte del co- 
mercio bilateral sea de productos primarios y de bajo valor agregado; de que las inversiones recíprocas son insuficientes; de que no haya suficiente cooperación y comercio en materia de ciencias y de tecnologías complejas; de que no haya conocimiento y entendimiento entre los diversos estratos sociales de ambos países, y de que los canales de información y de inversión, no sean muy expeditos, todo lo cual hace que las relaciones económicocomerciales actuales no puedan mantenerse en el mismo nivel de cooperación.

\section{El Tratado de Libre Comercio entre China y Chile se firmó en noviembre de 2005, tras cinco rondas de negociaciones.}

Teniendo presente la situación actual del mundo y de la región del Pacífico, así como las relaciones chino-chilenas, las autoridades de ambos países consideraron que era importante y necesario establecer mecanismos económico-comerciales entre ellos, y en junio de 2002, la República Popular China propuso a Chile iniciar negociaciones para un tratado de libre comercio. De acuerdo con ello, en abril de 2004, se iniciaron los estudios de factibilidad pertinentes y el 18 de noviembre de ese año, durante la cumbre del APEC realizada en Chile, los presidentes de Chile y de China proclamaron en forma conjunta el comienzo de las negociaciones para la celebración de dicho tratado. Tras cinco rondas de negociaciones, el 18 de noviembre de 2005 se firmó oficialmente el instrumento.

\section{LA FiRMA del Tratado de Libre Comercio entre China y Chile ha SIDO RESULTADO DE LAS NECESIDADES CONJUNTAS.}

Como país en desarrollo y con un gobierno de tendencia política centro-izquierdista, Chile ha estado aplicando una política exterior muy activa y multidimensional, orientada a establecer un orden internacional más justo y razonable, resistir el monopolio de los asuntos internacionales por las grandes potencias, y salvaguardar de forma inquebrantable la soberanía de los países en desarrollo. A partir de la recuperación del sistema democrático, se esfuerza en promover cambios y apertura, y desde el punto de vista geográfico, se centra cada vez más en la región del Pacífico. Al mismo tiempo, desde la década de 1990, junto con promover la reforma y el reajuste de las estructuras económicas y comerciales, Chile reconoce cada vez más la importancia de la zona del Pacífico. A su juicio, el siglo 21 será el siglo de Asia y el Pacífico, por lo cual considera que el afianzamiento de las relaciones con esa región le permitirá lograr crecimiento durante varios decenios. En los campos político, académico y empresarial, se estima importante participar en el círculo económico de la región AsiaPacífico. Desde hace tiempo, Chile aplica una política de no participar en grupos regionales excluyentes, sino promover activamente mecanismos bilaterales, con lo cual se sitúa en una posición favorable y ventajosa en lo económico-comercial, ya que de esta manera puede manejar y de- 
sarrollar múltiples conductos, formas y posibilidades de esta clase de relaciones. Asia, y especialmente Asia oriental ha sido desde años objeto de la atención de Chile, que destina la tercera parte de sus exportaciones a esa región. Como miembro oficial de APEC desde 1994, junto con Singapur, Chile propuso el programa del Foro de Cooperación América LatinaAsia oriental, que ha cumplido una función muy importante en la promoción de consultas y de cooperación entre los diversos países de la región.

\section{Chile destina la tercera parte de sus exportaciones al Asia oriental.}

En abril de 2004 entró en vigor el Tratado de Libre Comercio entre Chile y Corea, y se realizan negociaciones para celebrar este tipo de tratado con Japón y Singapur. Chile ha prestado creciente importancia a sus relaciones con China, ya que ambos tienen muchos puntos de vista comunes respecto de una serie de importantes temas internacionales y están igualmente empeñados en promover el desarrollo económico y social. Para Chile «tenemos la oportunidad histórica de crear una relación preferencial con China, con nítidas ventajas comerciales y de inversión. La inversión china en ultramar es un fenómeno emergente y partirá por socios que le sean altamente confiables. Chile califica bien en esa prueba, lo que podría ubicarnos en el epicentro del dinamismo de la economía global. Todo eso favorecería redes de negocios y alianzas tecnológicas y sectoriales (cobre, maderas) que estimularán nuestra capacidad de crecimiento por décadas, incluyendo la exploración conjunta de oportunidades en terceros mercados» (Declaración formulada por Osvaldo Rosales, Director General de Relaciones Económicas Internacionales de la Cancillería de Chile, publicada en La Tercera, el 17 de septiembre de 2004) y confía en estrechar las relaciones económico-comerciales con China y en especial establecer mecanismos eficientes para participar aun más en el proceso de crecimiento económico de China y obtener beneficios en materia de comercio y de inversión. En las negociaciones del tratado de libre comercio, la parte chilena demostró comprender bien la importancia de las relaciones bilaterales y de aprovechar las ricas experiencias y la alta eficiencia administrativa de su contraparte.

Mientras que en las edades moderna y contemporánea China, el país en desarrollo de mayor tamaño, fue muy pobre, atrasado y humillado por las grandes potencias del mundo, y reiteradamente perdió oportunidades de desarrollo, en 1978 inició la aplicación de políticas de reforma y apertura, aprovechando las oportunidades de desarrollar la economía, aumentar las fuerzas del país y elevar el nivel de vida de las masas populares y hacer de China uno de los países más poderosos del mundo, objetivos comunes de toda la nación china. China está convencida de que enfrenta las oportunidades de desarrollo más importantes de su historia y confía en que habrá 20 años más de paz mundial, lo que considera una valiosa oportunidad estratégica, lo que la mueve a contribuir a salvaguardar dicha paz y estabili- 
zar la situación regional y mundial. China sostiene «la política exterior de independencia, autonomía y de paz», se esfuerza por arreglar bien sus relaciones con las grandes potencias del mundo, $\mathrm{y}$ al mismo tiempo, se preocupa por desarrollar lo más posible las relaciones amistosas y de cooperación con los países vecinos y con los países en desarrollo de África y América Latina. Con este fin está dispuesta a promover, con su desarrollo, el crecimiento económico de los países en desarrollo del mundo, y espera también beneficiarse de la cooperación con el mundo en desarrollo.

\section{Desde hace tiempo la economía de Chile ha evolucionado en forma muy favorable.}

Las relaciones entre China y los países de América Latina se han desarrollado muy rápidamente desde los años 1990. Ha establecido relaciones importantes y eficientes de cooperación e intercambio con los mecanismos regionales de América Latina, incluidos el Grupo del Río, el Parlamento Latinoamericano, la OEA, el Mercosur, la Comunidad Andina, la CARICOM, etc., y confía en profundizarlas aún más con los diversos países latinoamericanos, y en especial establecer con ellos mecanismos eficientes de cooperación económica y comercial.

Desde hace tiempo, la economía de Chile ha estado evolucionando muy bien. Su crecimiento es sostenido, estable y relativamente acelerado; su situación político-social es estable; en el campo político, las cosas marchan en la dirección ade- cuada; el marco legal es bastante perfecto y eficiente; el reajuste macroeconómico se aplica en forma equilibrada; su política exterior es activa y sus relaciones con el exterior muy equilibradas; ha adquirido una rica experiencia en la negociación de tratados de libre comercio. Desde el punto de vista geográfico, Chile ha dado un importante paso para la comunicación entre Asia oriental y América Latina; con una economía no muy grande pero internacionalmente muy competitiva, su experiencia histórica de promoción de relaciones amistosas y de cooperación con China es muy positiva; su convicción de participar en el desarrollo de China y de Asia oriental y el Pacífico, lo convierten en la mejor opción para China en América Latina. El tratado de libre comercio entre China y Chile podrá servir de referencia y de ejemplo para las relaciones de China con los demás países latinoamericanos.

\section{El tratado de libre COMERCiO entre China y ChILE va a PROMOVer CON FUERZA EL DESARROLLO DE RELACIONES BILATERALES.}

El tratado de libre comercio entre ambos países, que entrará en vigor el segundo semestre de 2006, se basa en la igualdad, la comprensión y la concesión recíproca de beneficios, constituye el mecanismo económico-comercial más importante suscrito entre ambos países, y sin duda alguna acelerará las relaciones económico-comerciales entre ambos. El tratado permitirá que las partes pongan ple- 
namente en juego sus respectivas ventajas, compensen las deficiencias de la otra, y resuelvan adecuadamente las controversias comerciales de modo de evitar conflictos, promover el desarrollo de diversos sectores de la economía e incrementar el empleo. El tratado favorecerá también el desarrollo de relaciones entre China y los países sudamericanos, así como aquellas entre Chile y los países asiáticos.

Cabe mencionar que la entrada en vigor del tratado de libre comercio entre China y Chile corresponde al primer año del «décimo primer programa quinquenal del desarrollo de la economía nacional y de la sociedad» de China, y según estimaciones oficiales, durante su ejecución, el crecimiento económico será en promedio de $8.5 \%$ anual, y hasta 2010 el PIB de China será del orden de 26,000 billones de Yuan de Renminbi (alrededor de 3,250 billones de dólares de Estados Unidos). La esencia moral del décimo primer programa quinquenal de China radica en la adquisición del concepto científico de desarrollo, fomentando el desarrollo armonioso, acortando las diferencias entre pobres y ricos, entre ciudades y campo y entre diversas regiones del país, para llegar a un desarrollo científico y razonable. Se promoverá la transformación del desarrollo económico de un modelo de promoción basado en las necesidades exteriores a otro de promoción de las necesidades internas, incluidos gastos e inversiones; la explotación de la zona occidental del país; la renovación de las viejas bases industriales del noreste; la construcción de infraestructura en todo el país; la erradicación de la pobreza de las masas miserables, así como el mejoramiento del nivel y de la calidad de vida de las masas populares. Todo ello puede originar enormes necesidades; además, las diversas regiones y provincias de China, con su variedad de costumbres, culturas y hábitos sociales, hacen que las necesidades sean muy variables, y den lugar a un amplio espacio de cooperación para las empresas chilenas.

\section{La variedad de costumbres, culturas y hábitos sociales de China da amplio espacio de cooperación para las empresas chilenas.}

\section{SugerEnCIAS Y PROPUESTAS}

Es indispensable aprovechar el creciente desarrollo de la globalización económica, de las nuevas ciencias y de las tecnologías complejas, de las nuevas modalidades de transferencia de información y de comunicación y transporte, y afianzar el intercambio político y entre los diversos sectores sociales de ambos países, a fin de profundizar el conocimiento y el entendimiento recíprocos. Habría que probar nuevas formas de cooperación, abrir nuevas áreas de cooperación y promover programas nuevos de cooperación.

$\mathrm{Al}$ respecto, habría que mantener intercambio entre los estratos políticos de ambos países, considerando la posibilidad de crear y reforzar mecanismos de diálogo, consulta y visitas mutuas, como ser mediante comisiones bilaterales, foros bi- 
laterales etc., e incrementar las consultas y el apoyo recíproco respecto de importantes asuntos internacionales en los mecanismos multilaterales del mundo.

En segundo lugar, habría que reforzar el intercambio entre diversos sectores sociales de ambos países para promover el conocimiento y el entendimiento entre los sectores académico, empresarial, cultural, popular, juvenil y de las organizaciones femeninas así como de los medios de comunicación. Valdría la pena considerar la posibilidad de incrementar las actividades culturales bilaterales y establecer centros culturales.

En tercer lugar, habría que iniciar y completar cuanto antes negociaciones sobre el comercio de servicios e inversiones, esforzándose por llegar a proyectos sobre medio ambiente, bienestar y movimientos de mano de obra y de recursos humanos, mutuamente aceptables para profundizar las relaciones en su conjunto y establecer un compromiso pleno entre ambos países. Además de los temas relacionados con el comercio de productos y de servicios, de inversión y mano de obra, habría que prestar más atención a afianzar la cooperación en las áreas económica, científica y tecnológica y cultural, a fin de ampliar las bases de los intereses conjuntos, y promover el mejoramiento y la renovación de las relaciones bilaterales.

Cuarto, al mismo tiempo de regular las relaciones económico-comerciales bilaterales con arreglo al tratado de libre co- mercio, habría que reforzar la cooperación bilateral con terceros países, a fin de ampliar los efectos de las relaciones bilaterales al resto de los países de América Latina y de Asia.

\section{Habría que reforzar el intercambio entre los diversos sectores sociales de ambos países.}

Quinto, deberían explorarse y probarse nuevos modelos, y formas de cooperación chino-chilenas, por ejemplo, considerando las diferentes especialidades regionales de ambos, incluso estructuras económicas, costumbres, y tradiciones, así como diferentes necesidades, promoviendo el intercambio de productos que requieren las diversas regiones y sectores económicos; reforzar múltiples modelos de cooperación, incluidos de inversión, coinversión, cooperación etc.; promover de forma cooperativa la construcción de infraestructura relacionada con las relaciones económico-comerciales chino-chilenas, incluidas las de otros países sudamericanos, a fin de que el efecto de cooperación se amplíe hacia otros países de la región.

Finalmente, deberían explotarse las potencialidades de las empresas privadas de China, de modo que participen en los esfuerzos por afianzar las relaciones chino-chilenas, incluidas las microempresas, la pequeña y la mediana empresa y las empresas de gran magnitud. 